\title{
WIRELESS SENSOR NETWORK BASED LOCALIZATION IN INDUSTRIAL ENVIRONMENTS
}

\author{
László Gogolák ${ }^{1}$, Igor Fürstner ${ }^{1}$, Szilveszter Pletl ${ }^{1,2}$ \\ ${ }^{1}$ Subotica Tech, Subotica, Serbia \\ ${ }^{2}$ University of Szeged, Department of Informatics, Szeged, Hungary, \\ gogolak@vts.su.ac.rs, ifurst@vts.su.ac.rs,pletl@inf.u-szeged.hu
}

\begin{abstract}
The use of wireless devices has greatly increased in the last decade, and it has been one of the most widely used medium of information transmission. Within the wireless devices the wireless sensor networks are the most contemporary and most commonly researched field. The work deals with the industrial use of wireless sensor networks and more precisely with monitoring and controlling industrial assembly lines. The focus of this study is localization by the use of wireless technology in the above mentioned environment. In the experiment wireless sensors are placed on the base elements of currently being assembled products. The developed system is able to specify the precise place of the product in the assembly line and record the time of localization. By the use these information the time of assembling the product can be monitored. For determining the place of the product the Received signal strength indication - RSSI has been used. The current position of the product is calculated by a neural network. The use of these sensors makes possible the measuring and recording of the influences on the product during the assembly, such as the effects of temperature, humidity, or if the product has been hit or damaged. By the use of these wireless sensor networks the quality of the assembled products can be improved and the process of assembly can be optimized.
\end{abstract}

Keywords: Fingerprint location, WSN, Received Signal Strength, Mobile sensor

\section{INTRODUCTION}

The outdoor localization is widely used thanks to the highly developed GPS. Numerous devices contain elements for receiving and processing GPS signs. One's position can be determined on each spot of the Earth with an accuracy of $2 \mathrm{~cm}$. To obtain this level of accuracy, we must possess the right devices and be in an open field since this type of localization can work only in an open field. As for the indoor localization, more solutions are possible by using different technologies. The most common way to determine an indoor position is the processing of an RF sign. This localization is called the fingerprinting method (Radu et al 2005, Mustapha et al 2009). This work presents a localization method which uses the Wireless Sensor Network WSN for indoor localization even in an industrial environment.

More precisely, the work presents the results of two experiments. During the first experiment WSN sensor modules were placed on permanent positions (anchor modules). The room was not of an ideal structure because it was equipped with furniture, computers, there were more windows and doors on the walls. With the help of a mobile WSN module measurements were done on certain spots of the room. The RSSI (Received Signal Strength Indicator) values were measured between the mobile and each anchor. These results were collected in a database, and were used to find some correspondence between the changes of the RSSI signs and the position 
of the mobile sensor by using neural networks (Kermani et al 2005). The given database was used for teaching a neural network which is then capable of determining the position of the mobile sensor based on a new measurement result. The neural network testing was done in a simulated environment. The second experiment is the repetition of the first one in an industrial environment. The experiment aimed to prove the effects on the accuracy of localization of the magnetic noise in industrial objects. The process of localization is an element of a system that is continuously following the position and the environmental effects on the products being assembled.

\section{WIRELESS SENSOR NETWORK}

Wireless sensor networks are useful because there is no need for wires and this provides the possibility to measure quantities which were not possible in the past. Measuring in highly explosive risk areas was a problem due to the potential levels of grounds. Highly corrosive vapours in the air damage the cabling of sensor networks requiring frequent maintenance. Wireless sensor networks are successfully used in forest fire detection systems, monitoring of agricultural microclimate, monitoring of traffic structures and their load (Gogolak et al 2010).

The Crossbow IRIS (Fig. 1) wireless sensors nodes compliant with IEEE 802.15.4 standard were used for measurement of the RSSI values. Crossbow technology has compact wireless modules with complete software support. The $2.4 \mathrm{GHz}$ modules have an 8bit low power microcontroller on-board and a wireless stage. The Atmel 1281 microprocessor is easy to program and has wide software support.

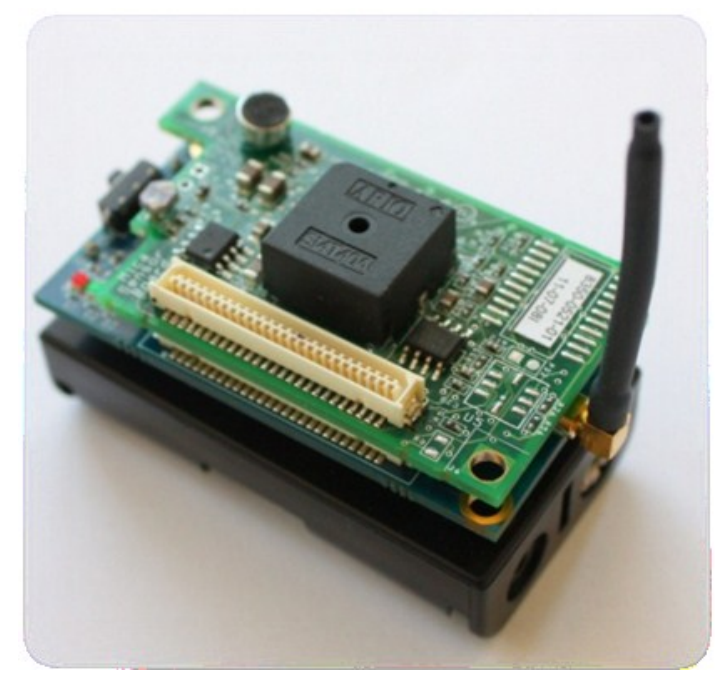

Figure 1 The Crossbow wireless mote

Crossbow provides complete source code for different types of wireless networks. The XMesh network stack is free, self organizing, provides support for low powered synchronizing of nodes, sleep modes. ZigBee and over-the-air programming are also supported. The radios provide a line of sight communication up to $500 \mathrm{~m}$ without an additional amplifier. The direct sequence spread spectrum radio which is resistant to RF interference and provides inherent data security. The XServe application is used to query the node's power states, consumed powers, SNRs, transmit powers and the topology of the self organized node network. A gateway is also used to gather the 
measurement data into a database. The modules are supported by TinyOS. TinyOS is an open source wireless sensor network system written in GCC, and is programmed in NesC.

\section{EXPERIMANTAL MEASURING AND DATABASE PREPARING}

Any localization algorithm needs a measurement database according to which it can determine a position. The bigger the database is, in other words the more information it contains, the more accurate the localization is. It is important to determine the target area, the room where the localization is needed. The structure, environment of the indoor space is an important factor which can affect the accuracy of the fingerprint localization, because it affects the quality of the wireless data transmit (Mao et al 2007, Gogolak et al 2011). The room where the measurements were done is a laboratory with tables, chairs and computers. The aim of the measurement and data collection was to be in a realistic environment. The most complicating factor during the data collection was the reflecting glass of the window, but it had an insignificant effect on the accuracy of the localization. A grid was set up in the room which contained $24 \times 10$ positions and had a resolution of 60 centimetres. In the experiment 5 fixed positioned WSN motes (Anchors) were used. The positions of the Anchors were optional, taking into consideration their equal distribution. The positions were placed in a distance of 60 centimetres from each other, and the RSSI values between the Anchor and the mobile mote were recorded on them. On each of the 5 Anchors 100 RSSI value samples were recorded. That means, 500 readings belong to each position. To obtain higher accuracy, the measurements were done in the other direction of the communication as well, to record the signal strength of the mobile mote in the Anchor motes. Since the grid consists of 240 positions, there are 12000 results in the mobile-anchor RSSI measurement and 12000 in the anchor-mobile RSSI measurement

After the recording of the data there are 24000 values in the database. The smallest RSSI value is 2 , while the biggest is 14 . This way we can differentiate 12 RSSI levels. This resolution is relatively small, and probably has a great effect on the accuracy of the localization, however, the Crossbow Iris sensor can measure signal strength only with this sensitivity. Since the environment of the room is not ideal, disturbances may occur in the wireless signal (János and Matijevics 2010). Fig. 2 shows the distribution of signal strength in the room for the anchor no. 3.

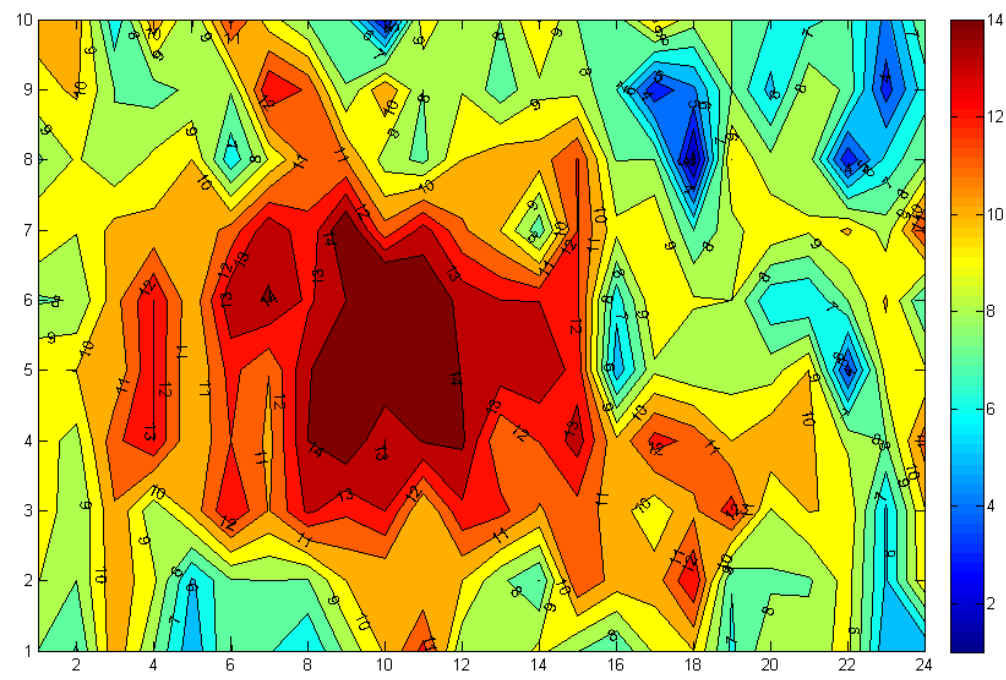

Figure 2 The RSSI values of anchor no. 3 for the every position 
Since some of the 100 samples are damaged due to loss of package, these are corrected with mean values. The data collected during the measurements will be used for the training of the neural network, so it is important that they are properly prepared. During the process we have worked with two databases. One contains the raw data, while the other contains the statistical values of the RSSI values for each position. In the latter the mean, median and standard deviation values of the RSSI values are shown.

\section{THE INDUSRIAL LABORATORY}

In the second phase of our experiment the process of localization was done in industrial environment, in an industrial laboratory equipped with an assembly line for assembling water pumps. This industrial laboratory provides the conditions of an industrial environment for studying wireless communication and localization processes (Toman et al 2009, Sárosi 2012). Fig. 3 shows the industrial laboratory.

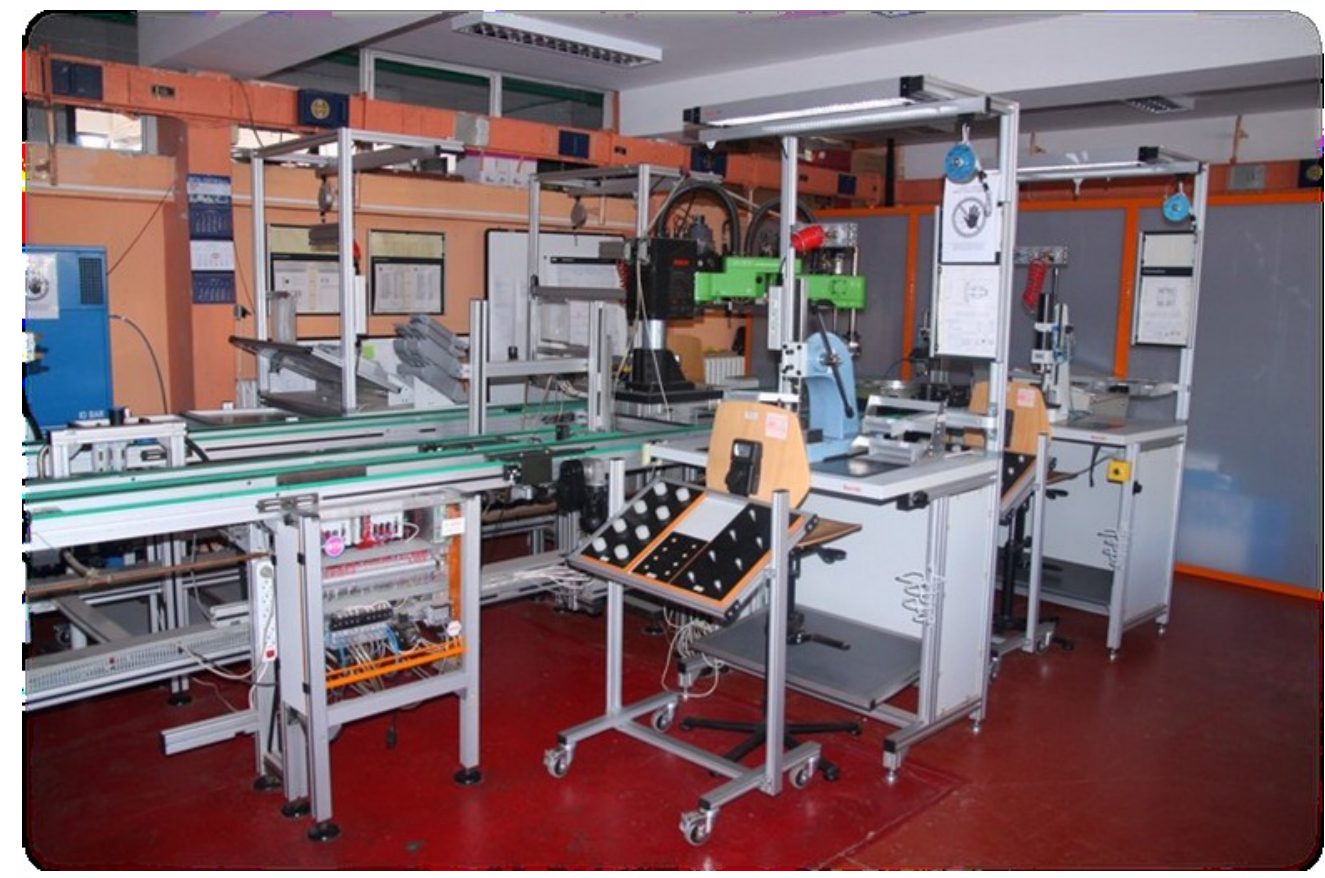

Figure 3 The industrial laboratory

The assembly line contains the following elements:

- Assembling stations

- Conveyor belt and palettes for conveying the base element

- Assembly devices

- Industrial BOSCH robot

In the presented laboratory setting up a wireless sensor system is necessary for localization. For successful localization it was also necessary to create the RSSI map of the assembly line. Finally, similarly to the test laboratory, in the case of the assembly line setting up anchors with fixed positions is needed. 
For creating the RSSI map the recording of the RSSI values is necessary between the anchors and the measuring sensor, which is done in characteristic measurement stations. The bigger the recordings of the RSSI values are, the better the quality of the RSSI map is. The RSSI values are saved in a database with the positions of the measurement stations. In case of the assembly line, the distance between the measurement points is $10 \mathrm{~cm}$.

\section{TEST RESULTS}

The aim of our work was to prove that the process of localization with the ,fingerprint" method can be realized in an industrial environment as well and can be applied with any production line or assembly line for developing different applications. For comparing the results the cumulative distribution function - CDF was used which shows the probability of the accuracy of localization. First the accuracy of localization was tested in those spots where the RSSI values were recorded. We selected some elements of the RSSI map as test values and they were studied with the help of the algorithm. Fig. 4 shows the CDF function for these "known" test dots.

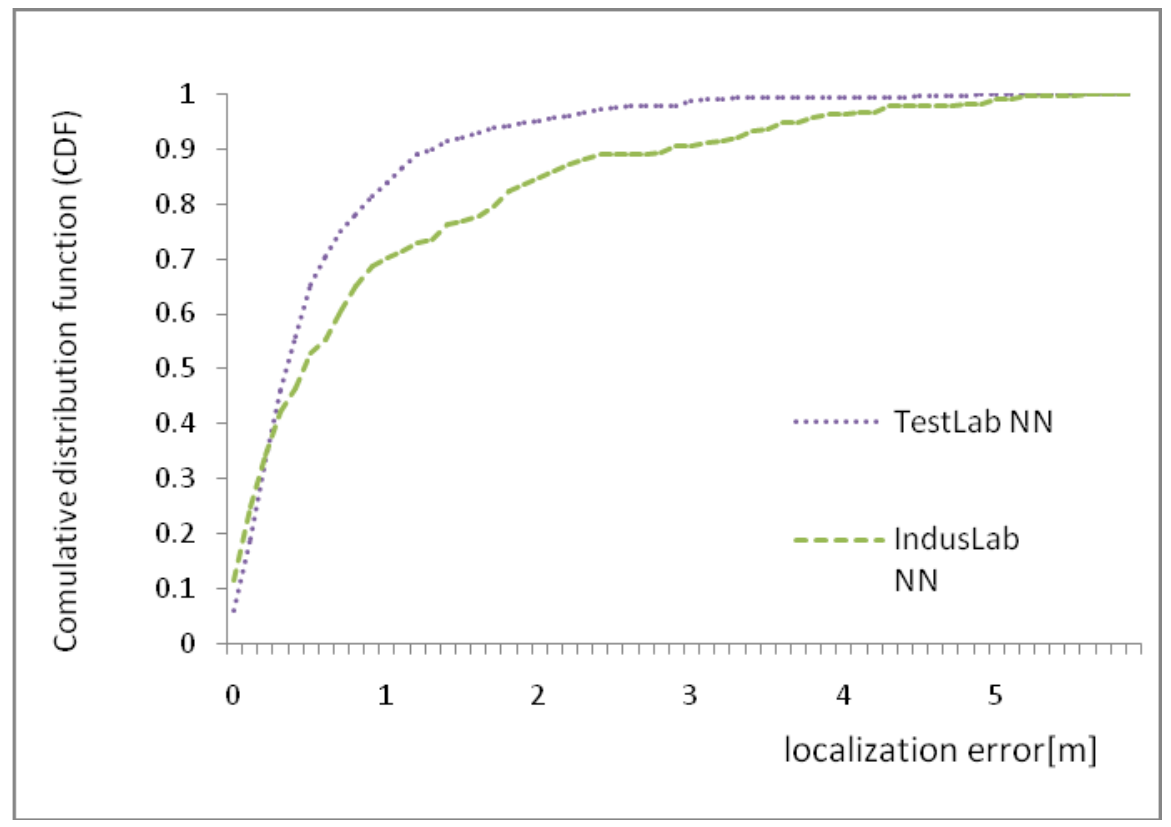

Figure 4 Probability distribution functions

As the curves on Fig. 4 suggest, the localization was more accurate in case of the test laboratory The bigger the rise of the curve is, the better the results are, which means that the localization is more probably precise. As it can be seen, the 0.5 metre accuracy can be determined with the same probability in both cases. After this the curves are distanced from each other. The accuracy on 1 metre can be determined with a 0.9 probability in case of the test laboratory, while in the industrial laboratory the probability is 0.7. Although the success of the localization in the industrial laboratory is markedly different from the test laboratory, it cannot be called unsuccessful. The accuracy on 2 metres can be determined with a probability of 0.9 , which is acceptable for industrial use. This result can be corrected by changing the structure of the sensor network and by the use of different localization algorithms. 


\section{CONCLUSION}

This work aimed to study the use of wireless sensor networks in industrial environment. We presented a ,fingerprint" localization process which makes indoor localization possible in a closed room. The experiment was done in both a test laboratory and in an industrial laboratory. A neural network was used for localization, and the accuracy of the localization was measured by a CDF function. The results show how the accuracy of the localization differs in case of a test laboratory from an industrial environment. According to the results the process of localization based on wireless sensor network can be pronounced successful. In our further studies we aim to apply the wireless sensor networks for monitoring and controlling of industrial assembly lines. In addition, we also aim to study the environmental effects on the product on the assembly line. We think that by developing a system like this the quality of the product and the manufacturing can be both improved.

\section{REFERENCES}

Stoleru, R., He, T., Stankovic, J. A., \& Luebke, D. (2005, November). A high-accuracy, low-cost localization system for wireless sensor networks. InProceedings of the 3rd international confenrence on Embedded networked sensor systems (pp. 13-26). ACM.

Boushaba, M., Hafid, A., \& Benslimane, A. (2009). High accuracy localization method using AoA in sensor networks. Computer Networks, 53(18), 3076-3088.

Gogolak, L., Pletl, Sz., Gál, P., Dukai, Z. (2010). Observing inland waters with ultrasonic distance measuring by the aid of wireless sensor networks" - SIP 2010 June 3-4, 2010 Subotica, Serbia XXVIII. "Science In Practice"

Mao, G., Fidan, B., \& Anderson, B. (2007). Wireless sensor network localization techniques. Computer networks, 51(10), 2529-2553.

Gogolak, L., Pletl, S., \& Kukolj, D. (2011, September). Indoor fingerprint localization in WSN environment based on neural network. In Intelligent Systems and Informatics (SISY), 2011 IEEE 9th International Symposium on(pp. 293-296). IEEE.

János, S., \& Matijevics, I. (2010, September). Implementation of potential field method for mobile robot navigation in greenhouse environment with WSN support. In Intelligent Systems and Informatics (SISY), 2010 8th International Symposium on (pp. 319-323). IEEE.

Kermani, Bahram G., Susan S. Schiffman, and H. Troy Nagle. (2005). Performance of the Levenberg-Marquardt neural network training method in electronic nose applications. Sensors and Actuators B: Chemical 110.1 13-22.

Toman P., Gyeviki J., Endrődy T., Sárosi J., Véha A. (2009). Design and Fabrication of a Testbed Aimed for Experiment with Pneumatic Artificial Muscle International Journal of Engineering, Annals of Faculty of Engineering Hunedoara, 2009, Vol. 7, No. 4, ISSN 15842665, pp. 91-94

Sárosi, J. (2012). New Force Functions for the Force Generated by Different Fluidic Muscles Transactions on Automatic Control and Computer Science, Scientific Bulletin of the „POLITEHNICA” University of Timisoara, 2012, Vol. 57 (71), No. 3, ISSN 1224-600X, pp. 135-140 